\title{
Commutative Algebra Provides a Big Surprise for Craig Huneke's Birthday
}

\author{
Irena Swanson \\ Communicated by Tom Garrity
}

A commutative algebra conference in July 2016 on the occasion of Craig Huneke's sixty-fifth birthday, included a major mathematical surprise.

Craig Huneke has been at the forefront of research in commutative algebra, introducing and advancing several influential notions, such as d-sequences, licci ideals,

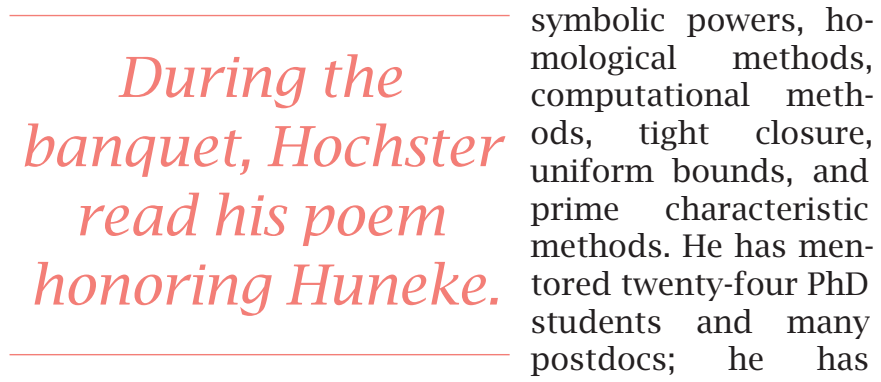
coorganized conferences, such as the Kansas-MissouriNebraska KUMUNU commutative algebra conference; he has served on the Executive Committee of the AMS and on the Board of Trustees of the MSRI.

Almost all the talks were directly related to Huneke's work, and most speakers started their talks describing how Huneke affected their work as a mathematician and as a person: they talked about his mathematical productivity, extensive collaborations, productive and precious lunches with napkin notes, excellent and influential talks, his advising, mentoring, his friendly competitiveness, and so on. Claudia Polini addressed how commutative algebra in general is friendly to women (possibly due to Emmy Noether being one of us) and in particular how Huneke has been a tremendous role model as a teacher, collaborator, mentor, and organizer. His own family life, with wife Edith

Irena Swanson is professor of mathematics at Reed College. Her e-mail address is i swanson@reed.edu.

For permission to reprint this article, please contact:

reprint-permission@ams.org.

DOI: http://dx.doi.org/10.1090/noti1480

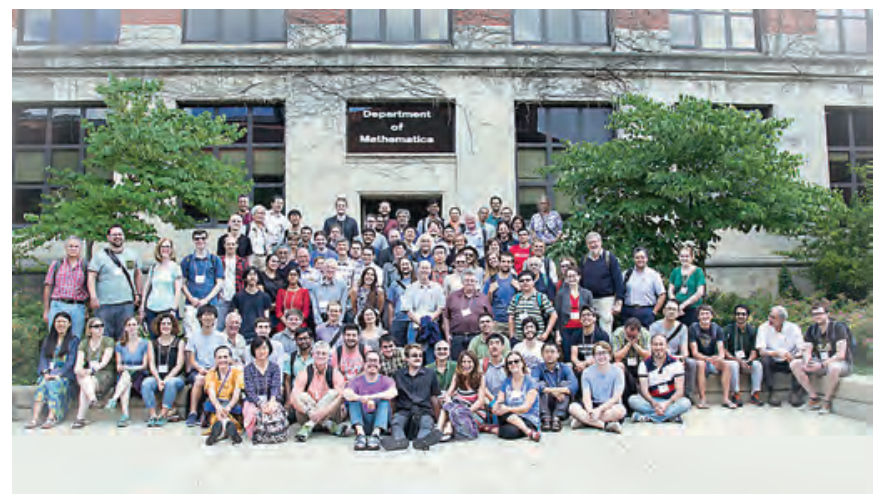

Group photo of the conference participants, Craig Huneke and Melvin Hochster in the center. Claudia Polini said that commutative algebra in general is friendly to women (possibly due to Emmy Noether being one of us) and in particular how Huneke has been a tremendous role model as a teacher, collaborator, mentor, and organizer.

Clowes, professor of Slavic languages and literatures, and their children Sam and Ned, has been a shining example for possibilities in home and professional life for women in academia. During the banquet, Hochster read his poem honoring Huneke. The most colorful roast was given by Hailong Dao, who mused how he had turned down a postdoc offer from the University of Kansas, where Huneke was based at the time, and when Hailong later joined the University of Kansas in a tenure-track position, Huneke "won" the postdoc standoff by leaving after Hailong's three years there. Neal Epstein, Karl Schwede, and I wrote a song that the whole room sang for Huneke to the tune of Woodie Guthrie's "This Land Is Your Land," one of Huneke's favorite songs. 


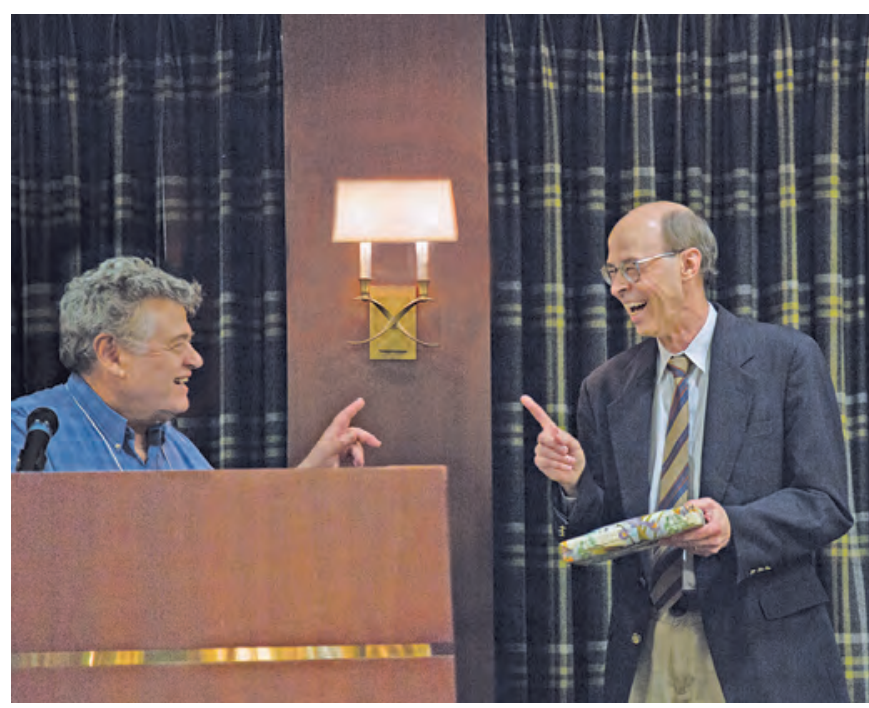

Melvin Hochster read his touching poem and presented his plaque to Craig Huneke.

There were twenty-nine talks over six days, but the really big deal was two consecutive talks on day five by Jason McCullough and Irena Peeva on Rees-like algebras. As an organizer I was aware that the two wanted to give consecutive talks, but I was not aware of their research collaboration, nor did their abstracts hint at anything field-changing. Whereas their talks were spectacular in content and in the changes they are bringing to the field, their abstracts were clever decoys, Peeva's just mentioning "some open questions."

It turns out that Rees-like algebras are very interesting and powerful and that the discussion of the open questions was the big deal!

McCullough started with an announcement that Peeva and he were coordinating their talks and tried hard to get them ready for the conference as a birthday surprise of a sort, which meant that the audience would have to come back for Peeva's talk to get the rest of the story. Early in his talk McCullough brought up the CastelnuovoMumford regularity (the smallest integer such that related cohomology modules vanish in shifted higher degrees) and some open questions in this area, such as the EisenbudGoto conjecture and the Bayer-Stillman conjecture. The audience was now abuzz: is the surprise about resolving one of these two? Which one? Or is it something else? Is the answer positive or negative?

The Eisenbud-Goto conjecture ([EG], 1984) estimates the Castelnuovo-Mumford regularity reg $(P)$ of a homogeneous prime ideal $P$ in a polynomial ring $S$, such as $\mathbb{C}\left[x_{1}, \ldots, x_{n}\right]$, in terms of the degree (multiplicity) of $S / P$ and the codimension of $P$ :

$$
\operatorname{reg}(P) \leq \operatorname{deg}(S / P)-\operatorname{codim}(P)+1 .
$$

Bayer and Mumford (1993) had discussed that the missing link between the sharp doubly exponential bound on regularity of arbitrary homogeneous ideals and the nice bounds on regularity in the smooth case is that

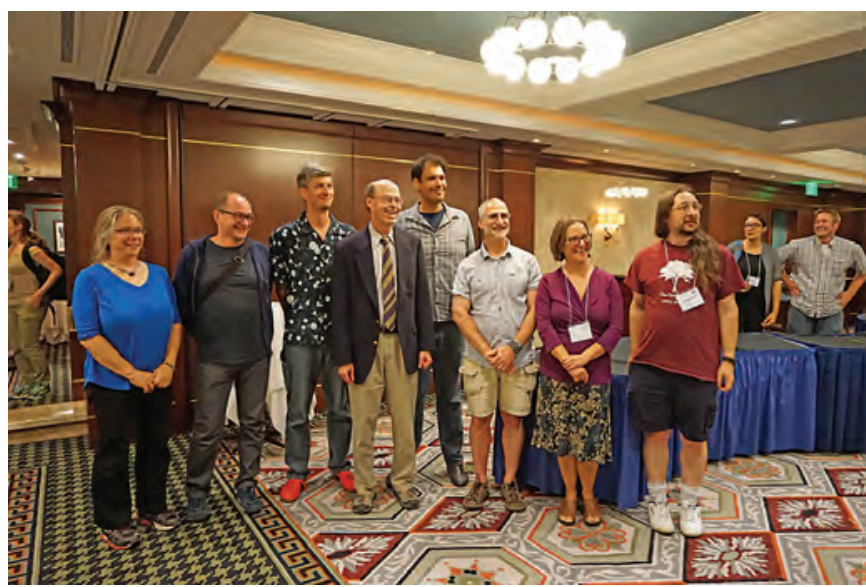

Huneke's postdocs, from left to right: Karen Smith, Alberto Corso, Graham Leuschke, (Craig Huneke), Luis Núñez-Betancourt, Ian Aberbach, Adela Vraciu, Jeff Mermin. Standing in the background: PhD students Janet Striuli and Branden Stone.

we do not yet have a decent bound on the regularity of all reduced equidimensional ideals. The Eisenbud-Goto Conjecture was aiming to fill in this gap. Special cases have been proved.

In their talks at the conference, McCullough and Peeva reported on their new techniques for handling projective dimension and Betti numbers: Rees-like algebras and step-by-step homogenization.

The classical Rees algebra of an ideal is a workhorse for analyzing powers of that ideal in one fell swoop, and the projective scheme of a Rees algebra is the blowing up of the spectrum of the ring along the subscheme defined by the ideal. Explicitly, the Rees algebra of an ideal $I$ in a commutative ring $S$ is a subring of the polynomial ring $S[t]$ in one variable $t$, and it is generated over $S$ by elements at as $a$ varies over $I$. The classical extended Rees algebra of $I$ is $S\left[I t, t^{-1}\right]$, which is generated over $S[I t]$ by $t^{-1}$. Huneke has done great work with Rees algebras, including analyzing them for ideals generated by d-sequences, determining their Cohen-Macaulay and integral closure properties, and exploring their analogues for modules with Eisenbud and Ulrich (2002).

McCullough and Peeva defined and developed a new Rees-like algebra $S\left[I t, t^{2}\right]$ of $I$, generated over $S[I t]$ by $t^{2}$. This notion is a generalization of a local example due to Hochster where he produced a family of prime ideals with fixed embedding dimension and fixed Hilbert-Samuel multiplicity but arbitrarily many minimal generators.

For the context of the conjectures, let $S$ be a polynomial ring over a field; let $I$ be an ideal in $S$ generated by homogeneous elements $f_{1}, \ldots, f_{m}$; let $T$ be the polynomial ring generated over $S$ by $m+1$ variables $y_{1}, \ldots, y_{m}, z$; let $\varphi: T \rightarrow S\left[I t, t^{2}\right]$ be the $S$-algebra map taking $y_{i} \mapsto f_{i} t$ and $Z \mapsto t^{2}$; and finally, let $Q$ be the kernel of $\varphi$. McCullough and Peeva proved the following:

1. $Q$ is a prime ideal (minimally) generated by the elements $\left\{y_{i} y_{j}-z f_{i} f_{j}: 1 \leq i, j \leq m\right\}$ and 


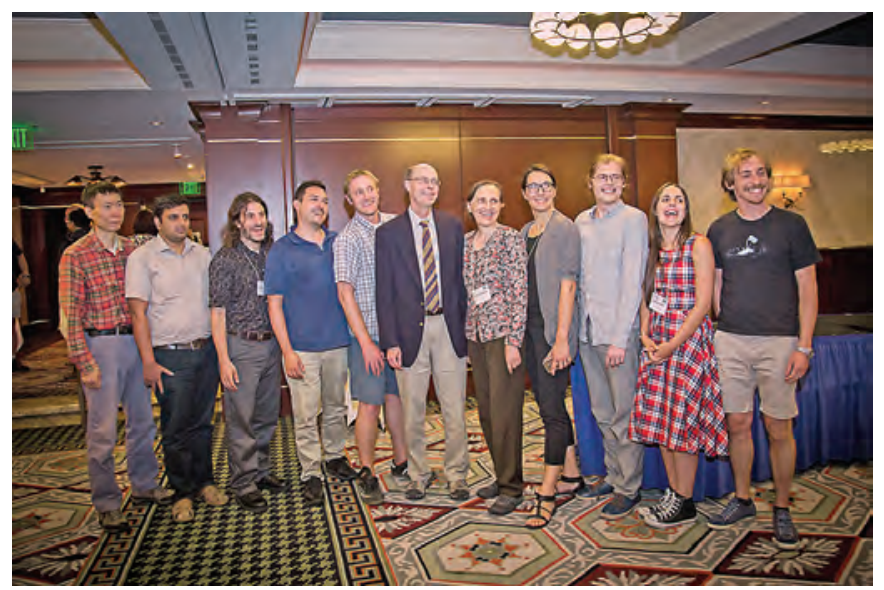

Huneke's PhD students at the conference, from left to right: Yongwei Yao, 2002; Manoj Kummini, 2008; Neil Epstein, 2005; Giulio Caviglia, 2004; Branden Stone, 2012; Craig Huneke; Irena Swanson, 1992; Janet Striuli, 2005; Ilya Smirnov, 2015; Elóisa Grifo, current student; Alessandro De Stefani, 2016.

$\left\{\sum_{i=1}^{m} c_{i j} y_{i}: \sum_{i=1}^{m} c_{i j} f_{i}=0\right\}$. Thus we have an explicit set of generators of $Q$, the latter kind arising from the first syzygies of $I$. (In contrast, the generators of the presenting ideal of the classical Rees algebras are not well understood at all.)

2. If we assign $\operatorname{deg}\left(y_{i}\right)=\operatorname{deg}\left(f_{i}\right)$ and $\operatorname{deg}(z)=2$, then $Q$ is a homogeneous ideal in $T$. Furthermore, if $\operatorname{deg}\left(f_{i}\right) \geq 2$ for all $i$, the graded Betti numbers of $T / Q$ over $T$ equal the graded Betti numbers of $T /(Q+(z))$ over $T /(z)$ since $z$ is a non-zerodivisor. These Betti numbers are well understood because the minimal free resolution of $T /(Q+(z))$ over $T /(z)$ is a mapping cone with ingredients being the minimal $S$ resolution of $I$ and the well-known Koszul resolution and the Eagon-Northcott resolution.

The only "problem" with Rees-like algebras is that the constructed polynomial ring $T$ is not standard graded and so not immediately relevant for resolving the EisenbudGoto and the Bayer-Stillman conjectures. The classical approach is to change the degrees of the variables to 1 and then homogenize the ideal. However, Betti numbers change under homogenization, and so all of the information about the Rees-like algebra would be lost in this way. McCullough and Peeva invented a way to remove the nonstandard obstacle, and they called their process step-by-step homogenization. They homogenize the ideal repeatedly, one variable at a time. The striking discovery is that this process preserves the Betti numbers if the ideal is prime, as it is in their case. In contrast to classical homogenization, where one needs to homogenize a Gröbner basis in order to obtain generators of the homogenized ideal, for the step-by-step homogenization it suffices to homogenize the generators of $Q$. Without going into too much detail, the homogenized $Q$ is a prime ideal in a standard graded polynomial ring, it has the same number of generators as $Q$, and its dimension, depth, projective dimension, Castelnuovo-Mumford regularity, and degree are all well understood and well behaved. For example, the homogenized prime's quotient has degree equal to $2 \prod_{i=1}^{m}\left(\operatorname{deg}\left(f_{i}\right)+1\right)$, and its projective dimension equals $m-1$ plus the projective dimension of $S / I$.

This is a beautiful and powerful construction, all by itself!

We got this far in the second talk, delivered by Peeva. We were close to the inevitable big announcement, but Peeva paused right there, leaving us in suspense a little longer, to address the honoree of the conference with high praise for his research, service, and enormous impact on the field. The context made her words all the more powerful.

And then she went for what their birthday surprise was all about. Namely, starting with an arbitrary homogeneous ideal in a polynomial ring $S$, via step-by-step homogenization of the defining ideal of its Rees-like algebra, one gets a prime ideal $P$ in a polynomial ring $S$ with precise control on the dimension of $S$, on the number of generators of $P$ and their degrees, on the dimension, depth, multiplicity, regularity, and projective dimension of $S / P$. In particular, if they start with the famous Mayr-Meyer ideals (1982) or variant ideals due to Koh (1998) and Bayer and Stillman (1988), the prime ideals constructed via this machinery yield counterexamples to the EisenbudGoto conjecture and the Bayer-Stillman conjecture. And worse, McCullough and Peeva showed that the regularity of reduced irreducible schemes is not bounded above by any polynomial in the degree. Peeva finished her talk with a discussion of compelling future redirections.

Peeva's and McCullough's lectures were masterfully delivered: they carried amazing content, and the suspense was just right. The two got huge applause.

Huneke, instead of the usual questions for the speakers, turned to the audience and said: "When I was seven years old, I wanted a bicycle. I really, really wanted a bicycle. And I got it for my birthday, and that was the best birthday present to me ever-until this present right now."

These results of McCullough and Peeva are reshaping the field of commutative algebra: their counterexamples to the Eisenbud-Goto conjecture not only show that the conjectured bound does not hold but also that there is no bound on the regularity of irreducible varieties that is a polynomial function of the degree. Furthermore, McCullough and Peeva produced a beautiful and general machinery that is worthy of deeper study, and in fact they are working with collaborators on further papers.

The summer of 2016 was amazing for commutative algebra: McCullough and Peeva resolved a thirty-twoyear-old problem, then Yves André proved the direct summand conjecture which was posed in its general form by Mel Hochster forty-seven years ago, Bhargav Bhatt significantly shortened the proof, and Tigran Ananyan and Mel Hochster gave a positive answer to Stillman's question. 
ACKNOWLEDGMENTS. I thank Melvin Hochster for the lion's share of the organization of the conference, including convincing Huneke that a conference was in order. I also thank Craig Huneke, Jason McCullough, Irena Peeva, Marilina Rossi, and Bernd Ulrich for feedback on early versions of this article, and Bernd Ulrich for sharing the slides of his talk.

\section{References}

[EG] D. EISENBUD and S. GoTo, Linear free resolutions and minimal multiplicity, J. Algebra 88 (1984), 89-133. MR 0741934

\section{Photo Credits}

Group photo of the conference participants is courtesy of Margie Morris.

Photo of Melvin Hochster reading his poem is courtesy of Margie Morris.

Photo of Huneke's postdocs is courtesy of Irena Swanson. Photo of Huneke's PhDs students at the conference is courtesy of Margie Morris.

Photo of Irena Swanson is courtesy of Irena Swanson.

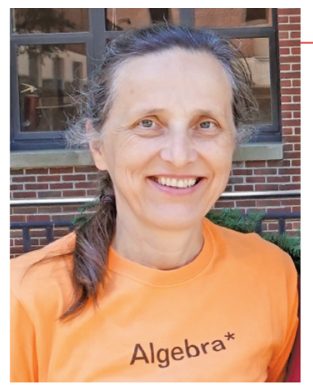

\section{ABOUT THE AUTHOR}

Irena Swanson sports the Algebra* T-shirt designed on the occasion of Craig Huneke's sixtieth birthday by Amelia Taylor, with help from Ananthnarayan Hariharan and Manoj Kummini.

Irena Swanson

\section{Celebrating}

\section{Women's History Month

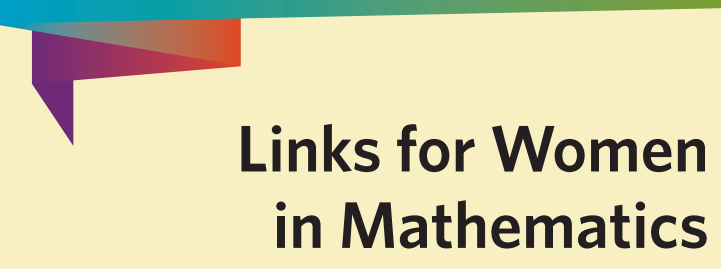

African Women in Mathematics Association:

africanwomeninmath.org/

Association for Women in Mathematics:

https://sites.google.com/site/awmmath/home

Association for Women in Science:

www.awis.org

European Women in Mathematics: www.europeanwomeninmaths.org/

IMU Committee for Women

in Mathematics:

www.mathunion.org/cwm

Women in Math Project: darkwing.uoregon.edu/ $\sim$ wmnmath/

2017 Women in Mathematics, Science, and Technology Conference: www.millersville.edu/wmsc/

2017 Workshop "WIN4 Women in Numbers 4":

www.birs.ca/events/2017/5-dayworkshops/17w5083

2017 Workshop "Women in Control: New Trends in Infinite Dimensions": www.birs.ca/events/2017/

5-day-workshops/17w5123

. 